\title{
MicroRNA 21 targets $B C L 2$ mRNA to increase apoptosis in rat and human beta cells
}

\author{
Emily K. Sims ${ }^{1,2,3}$ • Alexander J. Lakhter ${ }^{1,2,3}$ • Emily Anderson-Baucum ${ }^{1,4}$. \\ Tatsuyoshi Kono $^{1,3,4}$ - Xin Tong ${ }^{1,4}$ - Carmella Evans-Molina ${ }^{1,3,4,5,6}$
}

Received: 21 December 2016 / Accepted: 3 February 2017 /Published online: 9 March 2017

(C) Springer-Verlag Berlin Heidelberg 2017

\begin{abstract}
Aims/hypothesis The role of beta cell microRNA (miR)-21 in the pathophysiology of type 1 diabetes has been controversial. Here, we sought to define the context of beta cell miR-21 upregulation in type 1 diabetes and the phenotype of beta cell miR-21 overexpression through target identification.

Methods Islets were isolated from NOD mice and mice treated with multiple low doses of streptozotocin, as a mouse model of diabetes. INS-1 $832 / 13$ beta cells and human islets were treated with IL- $1 \beta$, IFN- $\gamma$ and TNF- $\alpha$ to mimic the milieu of early type 1 diabetes. Cells and islets were transfected with miR-21 mimics or inhibitors. Luciferase assays and polyribosomal profiling (PRP) were performed to define miR-21-target interactions.
\end{abstract}

Electronic supplementary material The online version of this article (doi:10.1007/s00125-017-4237-z) contains peer-reviewed but unedited supplementary material, which is available to authorised users.

Emily K. Sims

eksims@iu.edu

1 Center for Diabetes and Metabolic Diseases, Indiana University School of Medicine, 635 Barnhill Drive, MS2031, Indianapolis, IN 46202, USA

2 Department of Pediatrics, Indiana University School of Medicine, Indianapolis, IN, USA

3 Herman B. Wells Center for Pediatric Research, Indiana University School of Medicine, Indianapolis, IN, USA

4 Department of Medicine, Indiana University School of Medicine, Indianapolis, IN, USA

5 Department of Biochemistry and Molecular Biology, Indiana University School of Medicine, Indianapolis, IN, USA

6 Richard L. Roudebush VA Medical Center, Indianapolis, IN, USA
Results Beta cell miR-21 was increased in in vivo models of type 1 diabetes and cytokine-treated cells/islets. miR-21 overexpression decreased cell count and viability, and increased cleaved caspase 3 levels, suggesting increased cell death. In silico prediction tools identified the antiapoptotic mRNA $B C L 2$ as a conserved miR-21 target. Consistent with this, miR-21 overexpression decreased BCL2 transcript and $\mathrm{B}$ cell lymphoma 2 (BCL2) protein production, while miR-21 inhibition increased BCL2 protein levels and reduced cleaved caspase 3 levels after cytokine treatment. miR-21-mediated cell death was abrogated in $828 / 33$ cells, which constitutively overexpress $B c l 2$. Luciferase assays suggested a direct interaction between miR-21 and the BCL2 3' untranslated region. With miR-21 overexpression, PRP revealed a shift of the $B c l 2$ message towards monosome-associated fractions, indicating inhibition of $B c l 2$ translation. Finally, overexpression in dispersed human islets confirmed a reduction in $B C L 2$ transcripts and increased cleaved caspase 3 production.

Conclusions/interpretation In contrast to the pro-survival role reported in other systems, our results demonstrate that miR-21 increases beta cell death via $B C L 2$ transcript degradation and inhibition of $B C L 2$ translation.

Keywords Animal-mouse - Basic science $\cdot$ Beta cell signal transduction $\cdot$ Cell lines $\cdot$ Islet degeneration and damage $\cdot$ Islets

$\begin{array}{ll}\text { Abbreviations } \\ \text { AOPI } & \text { Acridium orange/propidium iodide } \\ \text { Atf4 } & \text { Activating transcription factor 4 } \\ \text { BCL2 } & \text { B cell lymphoma 2 } \\ \text { BiP } & \text { Binding immunoglobulin protein } \\ \text { Chop } & \text { C/EBP homologous protein } \\ \text { ER } & \text { Endoplasmic reticulum } \\ \text { GSIS } & \text { Glucose-stimulated insulin secretion }\end{array}$




$\begin{array}{ll}\text { LNA } & \text { Locked nucleic acid } \\ \text { miR-21 } & \text { miRNA 21 } \\ \text { miRNA } & \text { microRNA } \\ \text { MLD-STZ } & \text { Multiple low-dose streptozotocin } \\ \text { PDCD4 } & \text { Programmed cell death 4 } \\ \text { PRP } & \text { Polyribosomal profiling } \\ \text { qPCR } & \text { Quantitative real-time PCR } \\ \text { ROS } & \text { Reactive oxygen species } \\ \text { STZ } & \text { Streptozotocin } \\ \text { UTR } & \text { Untranslated region }\end{array}$

\section{Introduction}

microRNAs (miRNAs) are short, non-coding RNAs that classically inhibit gene expression by increasing mRNA degradation or directly inhibiting mRNA translation [1]. Many studies have identified miRNAs as key regulators of beta cell development, glucose-stimulated insulin secretion (GSIS) and beta cell dysfunction [2-10]. Global expression profiling performed in human and rodent islets subjected to proinflammatory cytokine stress and viral infection as models of type 1 diabetes has demonstrated marked changes in miRNA expression patterns $[6,7,11]$. These findings suggest that beta cell miRNAs may also contribute to the activation of intrinsic beta cell stress pathways that act to augment or even initiate beta cell dysfunction and death during the development of type 1 diabetes [12]. miRNA 21 (miR-21) is an NFkB-dependent miRNA that has been shown to be induced during the evolution of type 1 diabetes [13-15]. This miRNA has been well characterised in other cell types, and has classically been labelled an 'oncomiR' in cancer cells, due to the inhibition of tumour suppressor genes, which produces pro-survival effects [16-18]. However, the role of miR-21 in beta cells has been less clear, with conflicting data regarding pro-survival vs proapoptotic effects $[13,14,19]$. Using human islets and cell line models of type 1 diabetes, we sought here to define the context of beta cell miR-21 upregulation and the phenotype of beta cell miR-21 overexpression by novel target identification. Our findings demonstrate an interaction between beta cell miR21 and the antiapoptotic mRNA $B C L 2$, resulting in dual effects on $B C L 2$ transcript abundance as well as $B c l 2$ translation.

\section{Methods}

Animals, islets and cell culture Eight-week-old male C57BL6/J mice (Jackson Laboratories, Bar Harbor, ME, USA) were treated with normal saline $(154 \mathrm{mmol} / \mathrm{l} \mathrm{NaCl})$ or streptozotocin (STZ) administered intraperitoneally at a dose of $35 \mathrm{mg} / \mathrm{kg}$ per day for 5 days to model type 1 diabetes. Blood glucose levels were measured via tail vein nick using an Alphatrak glucometer (Abbott Laboratories, Abbott Park,
IL, USA). Pancreatic islets were isolated by collagenase digestion 1 week after initiation of STZ, as previously described [20]. Eight-week-old female NOD mice (Jackson Laboratories), also used as an animal model of type 1 diabetes, were followed weekly for the onset of diabetes (blood glucose $>11.1 \mathrm{mmol} / \mathrm{l}$ ), at which point islets were isolated for analysis. Non-diabetic NOD mice were followed until 20 weeks of age to rule out the development of diabetes and then euthanised for islet isolation. Islets were also isolated from age-matched CD1 mice (Jackson Laboratories). Animals were maintained within the Indiana University Laboratory Animal Resource Center under pathogen-free conditions and protocols approved by the institutional animal care and use committee, in accordance with the Guide for the care and use of laboratory animals [21].

INS-1 832/13 and 828/33 cells were obtained from C. Newgard, Duke University, NC, USA and are commonly used by many investigators in the field [22, 23]. To ensure reproducibility, we adhere to standard culture techniques between all laboratory members and experiments. Cells were only used between passages 8 and 30 and the authenticity of the cell line was verified through maintenance of glucose-stimulated insulin secretion. INS-1 mycoplasma testing was performed to ensure negative status. INS-1 832/13 and 828/33 cells or cadaveric human islets from non-diabetic donors (obtained from the Integrated Islet Distribution Program, exempt from Institutional Review Board approval) were cultured as described $[23,24]$ and treated with a cytokine mix consisting of $5 \mathrm{ng} / \mathrm{ml} \mathrm{IL}-1 \beta, 100 \mathrm{ng} / \mathrm{ml} \mathrm{IFN}-\gamma$ and $10 \mathrm{ng} / \mathrm{ml} \mathrm{TNF}-\alpha$ for 6 , 24 or $48 \mathrm{~h}$. Cells were also treated with high glucose $(25 \mathrm{mmol} / \mathrm{l})$ or tunicamycin $(300 \mathrm{nmol} / \mathrm{l})$ for $24 \mathrm{~h}$.

Transfection INS-1 $832 / 13$ and $828 / 33$ cells were seeded into a 12-well plate at a density of $4 \times 10^{5}$ cells/well and treated for $48 \mathrm{~h}$ with miR-21 5p (accession no. MI0000569) mimic used at a concentration of $45 \mathrm{pmol}$ (Qiagen, Düsseldorf, Germany), or 100 pmol locked nucleic acid (LNA) inhibitor (Exiqon, Vedbaek, Denmark), or negative controls (Qiagen, Exiqon) that had been complexed with $3 \mu \mathrm{l}$ Lipofectamine 3000 and $100 \mu \mathrm{l}$ Opti-MEM (both from Thermo Fisher, Grand Island, NY, USA). Relative miR-21 expression after transfection is shown in the electronic supplementary material [ESM] Fig. 1.

For human islet dispersion and transfection, after $4 \mathrm{~h}$ of routine incubation at $37^{\circ} \mathrm{C}$, islets were suspended in $4 \mathrm{ml}$ of Accutase solution (Millipore, Billerica, MA, USA) with 100 units of DNase I (Millipore) in a thermal mixer at $37^{\circ} \mathrm{C}$, $1000 \mathrm{rev} / \mathrm{min}$ for $10 \mathrm{~min}$, followed by the addition of $10 \mathrm{ml}$ of culture medium. Dispersed cells were collected by centrifugation at $200 \mathrm{~g}$ for $3 \mathrm{~min}$, and then resuspended in culture medium and seeded into 12-well tissue culture plates (Falcon, Tewksbury, MA, USA). Dispersed cells from 200 islets were transfected with 23 pmol of either negative control miRNA or miR-21 mimic using Lipofectamine 3000 reagent as described above. Cleaved caspase 3 ELISA (ThermoFisher) was 
performed on transfected human islets as per the manufacturer's protocol.

Reporter assays Luciferase assays were performed using a Gaussia luciferase/secreted alkaline phosphatase dual reporter system (GeneCopoeia, Rockville, MD, USA). Plasmids containing the cloned wild-type human BCL2 3' untranslated region (UTR) or a mutated 3' UTR (positions 710-716 or 720-726) downstream of a secreted Gaussia luciferase reporter, driven by an SV40 promoter, and a secreted alkaline phosphatase reporter driven by a CMV promoter were generated by GeneCopoeia. INS-1 cells were seeded in a 12-well plate and then treated with $1 \mu \mathrm{g}$ of plasmid DNA complexed with $3 \mu \mathrm{l}$ of Lipofectamine 3000 (in $100 \mu \mathrm{l}$ Opti-MEM) for $24 \mathrm{~h}$. Cells were then trypsinised and reseeded, followed by transfection with a miR-21 mimic or negative control, as above, for $24 \mathrm{~h}$. A dual reporter luciferase assay kit (GeneCopoeia) was used according to the manufacturer's instructions to quantify luciferase activity in $10 \mu \mathrm{l}$ of medium. Results were normalised to secreted alkaline phosphatase activity measured using a SpectraMax M5 multiwell plate reader (Molecular Devices, Sunnyvale, CA, USA).

Cell counting and immunofluorescence Cell counting was performed using $20 \mu \mathrm{l}$ of trypsinised and resuspended INS-1 cells on a Cellometer (Nexcelom Bioscience, Lawrence, MA, USA). Live/dead staining was performed with acridium orange/propidium iodide dye (AOPI; Nexcelom Bioscience) mixed 1:1 with suspended cells after trypsinisation. Fluorescence detection of the generation of reactive oxygen species (ROS) in INS-1 cells was performed using CellROX Deep Red Reagent (ThermoFisher) as per the manufacturer's instructions. Cells were imaged on a LSM 700 fluorescent confocal laser scanning microscope (Zeiss, Göttingen Germany), and fluorescence intensity was assessed as mean signal per well using ZEN 2011 image processing software (Zeiss).

Quantitative real-time PCR RNA isolation and reverse transcription was performed using miRNeasy and miScript II RT kits according to the manufacturer's instructions (Qiagen). Quantitative real-time PCR (qPCR) was performed using the miScript SYBR Green PCR Kit (Qiagen) and a Mastercycler ep realplex instrument (Eppendorf, Hauppage, NY, USA). Relative RNA levels were established against the invariant small nuclear RNA RNU6-1 for miRNAs and $\beta$-actin for mRNA species, using the comparative $C_{t}$ method [25]. RNA primer sequences are listed in ESM Table 1.

Immunoblotting Immunoblot analysis was performed as previously described [26]. Bound primary antibodies were detected with donkey anti-mouse or donkey anti-rabbit antibodies. Antibody characteristics are detailed in ESM Table 2.
Immunoreactivity was visualised using fluorometric scanning on an Odyssey imaging system and quantified by LI-COR software (both from LI-COR Biotech, Lincoln, NE, USA).

Functional and translation assays GSIS was assayed as previously described [27]. Supernatants were collected and assayed using a radioimmunometric assay for insulin (Millipore). Values were normalised to the total insulin content of the islet fraction. Polyribosomal profiling (PRP) experiments were performed on INS-1 cell lysates as previously described [28]. Total RNA was reverse transcribed and qPCR was performed using SYBR Green methodology as above.

Statistical analysis Statistical analyses were performed using GraphPad Prism Version 6.00 (GraphPad Software, La Jolla, CA, USA). Student's $t$ tests were used for comparison between the treatment and control groups. One-way ANOVA with Tukey's post test for multiple comparisons was used when comparing more than two groups. For all analyses, a $p$ value of $\leq 0.05$ was considered significant.

\section{Results}

In order to determine whether inflammation present during the development of type 1 diabetes was responsible for induction of beta cell miR-21, several models of type 1 diabetes were utilised. Islets were isolated from two in vivo mouse models of type 1 diabetes: mice treated with multiple low-dose STZ (MLD-STZ) (obtained 1 week after treatment initiation) and NOD mice (at the time of onset of diabetes). Mice treated with MLD-STZ had increased islet miR-21 expression relative to saline-injected controls (Fig. 1a), while islets from diabetic NOD mice demonstrated an even more pronounced increase in miR-21 expression compared with CD1 controls (Fig. 1b). Interestingly, islets from non-diabetic NOD mice also exhibited increased miR-21 expression. Next, INS-1 832/13 cells were treated with a cytokine mix for 6,24 and $48 \mathrm{~h}$, and significantly increased miR-21 expression was observed at all time points (Fig. 1c-e). To define whether this effect on miR-21 could be related to other components of the diabetic milieu, INS-1 cells were treated with $25 \mathrm{mM}$ glucose, to induce glucotoxicity and oxidative stress, or $300 \mathrm{~nm}$ tunicamycin for $24 \mathrm{~h}$, to induce endoplasmic reticulum (ER) stress (Fig. 1f, g). Verification of treatment effects are shown in ESM Fig. 2. Neither condition increased miR-21 expression, suggesting that miR-21 was upregulated specifically in response to proinflammatory signalling and not in response to hyperglycaemia or ER stress.

To define the phenotypic effects of increased miR-21 expression, INS-1 $832 / 13$ beta cells were transfected with an miR-21 mimic. After 48 h, INS-1 cells with miR-21 

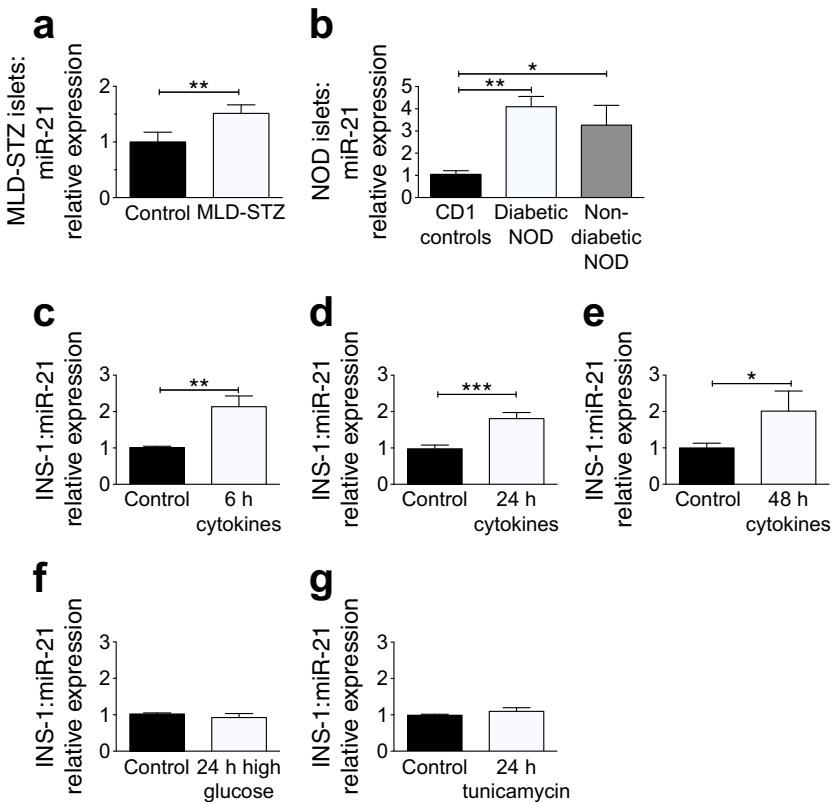

Fig. 1 Beta cell miR-21 production is induced in models of inflammation and type 1 diabetes. miR-21 expression was analysed in: islets from (a) mice treated with MLD-STZ or controls treated with saline $(n=5)$ or (b) diabetic and non-diabetic NOD mice ( $n=7, n=3$, respectively) compared with CD1 controls $(n=5),(\mathbf{c}-\mathbf{e})$ INS-1 cells treated with cytokine mix for 6,24 or $48 \mathrm{~h}(n=5-9)$, and (f) INS-1 cells treated with high glucose $(25 \mathrm{mmol} / \mathrm{l})$ or $(\mathbf{g})$ tunicamycin $(300 \mathrm{nmol} / \mathrm{l})$ for $24 \mathrm{~h}(n=5-6) . * p \leq 0.05$, $* * p \leq 0.01, * * * p \leq 0.001$

overexpression were significantly less confluent than cells transfected with a negative control (Fig. 2a, b), prompting live/dead staining with AOPI (Fig. 2a, c). AOPI staining revealed a significant increase in the percentage of dead INS-1 cells in response to miR-21 overexpression. To ascertain whether activation of intrinsic death pathways was involved, immunoblotting for cleaved caspase 3 was performed (Fig. 2d). A significant increase in cleaved caspase 3 protein levels confirmed a role for apoptosis in this phenotype. To explore activation of other intrinsic stress pathways, we quantified the expression of ER-stress-associated mRNAs in transfected cells. Interestingly, reduced expression of mRNAs encoding C/EBP homologous protein (Chop), the binding immunoglobulin protein $(\mathrm{BiP}) /$ glucose regulated protein 78, and activating transcription factor 4 (Atf4) (Fig. 2e) was observed. To assay for the presence of oxidative stress, we performed staining for ROS generation and were unable to detect any differences in transfected cells (Fig. 2f), suggesting that miRNA-21 decreased cell survival independently of ER and oxidative stress. To understand the effect of miR-21 upregulation on beta cell function, GSIS was performed in INS-1 cells transfected with a miR-21 mimic. In contrast to cells transfected with the negative control miRNA, INS-1 cells overexpressing miR-21 showed a blunted insulin secretory response to stimulation with high glucose concentrations (Fig. 2g).
Because the proapoptotic mRNA of the gene encoding programmed cell death 4 (PDCD4) has previously been confirmed as a target of beta cell miR-21, immunoblotting for PDCD4 was performed. Our results confirmed reduced PDCD4 protein levels, consistent with effective miR-21 overexpression (Fig. 3a) [19]. However, our observation that miR-21 overexpression increased beta cell death was at odds with a predicted pro-survival effect of reduced PDCD4. Based on this, we turned to in silico prediction tools to identify other potential miR-21 targets to explain the proapoptotic phenotype of beta cell miR-21 overexpression [29, 30]. We found that the 3' UTR of the mRNA encoding the antiapoptotic protein B cell lymphoma 2 (BCL2) was a predicted target for miR-21, with a predicted binding site that was well conserved among vertebrate species $[30,31]$. Figure $3 b$ depicts predicted sites of interaction between the human BCL2 3' UTR and miR-21. Identical consequential pairing regions are predicted at position 704-710 of the mouse Bcl2 $3^{\prime}$ UTR and 699-705 of the rat Bcl2 $3^{\prime}$ UTR [31].

Supporting this prediction, BCL2 protein levels were reduced in parallel with increased miR-21 levels after $48 \mathrm{~h}$ of cytokine treatment (Fig. 3c). To verify this relationship, qPCR was performed to assess relative $B c l 2$ transcript quantity on INS-1 832/13 cells transfected with a miR-21 mimic (Fig. 3d). This analysis revealed decreased transcript levels, consistent with mRNA degradation. In contrast, no significant change in the abundance of transcripts of other BCL2 family members that would be expected to impact apoptosis were detected (Fig. 3e). To determine whether a direct interaction occurred between miR-21 and the BCL2 $3^{\prime}$ UTR, INS-1 cells were transfected with plasmids containing a luciferase reporter driven by the wild-type human BCL2 3' UTR, or BCL2 3' UTRs that had been mutated at two different sites included in the predicted miR-21 binding region, positions $710-716$ or positions 720-726 (Fig. 3f). Cells were then transfected with either the miR-21 mimic or a negative control. Consistent with a direct interaction of miR-21 and the BCL2 $3^{\prime}$ UTR, miR-21 overexpression reduced the luciferase activity of INS- 1 cells expressing the wild-type $B C L 23^{\prime}$ UTR plasmid. However, no effect of miR-21 overexpression was present in INS-1 cells transfected with mutated 3' UTR plasmids.

To confirm a miR-21-mediated effect on protein levels, immunoblots for BCL2 protein were performed on INS-1 cells after miR-21 mimic transfection. This analysis demonstrated reduced levels of BCL2 protein with miR-21 overexpression (Fig. $3 \mathrm{~g}$ ). To understand whether the miR-21mediated reductions in BCL2 protein occurred exclusively through mRNA degradation as shown in Fig. $3 d$ vs direct inhibition of translation, PRP experiments were performed to ascertain the translation status of Bcl2 mRNA. Although no global changes in mRNA translation were seen (Fig. 3h), INS- 1 cells overexpressing miR-21 revealed a shift of $B c l 2$ transcripts towards monosome-associated fractions, and away 


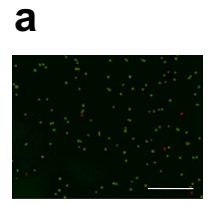

Negative control

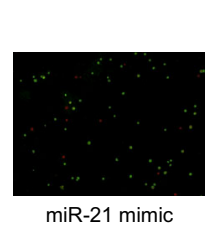

d

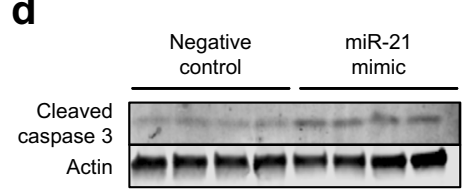

Actin
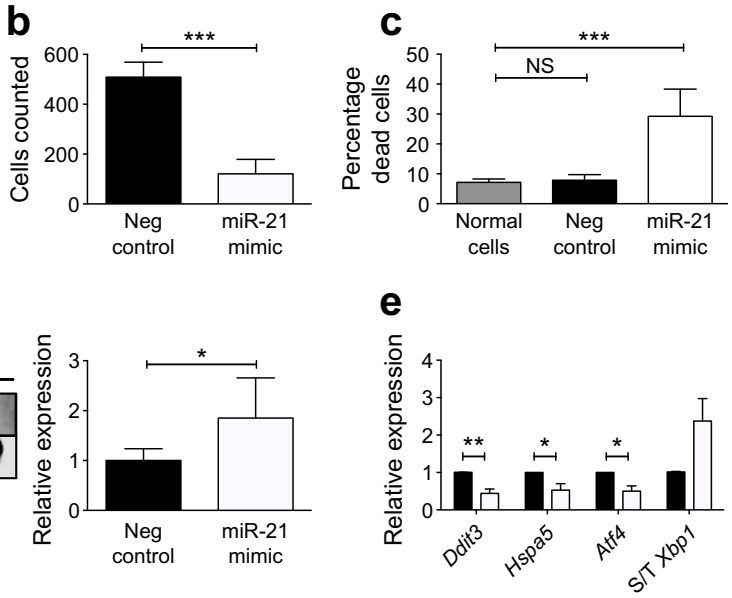
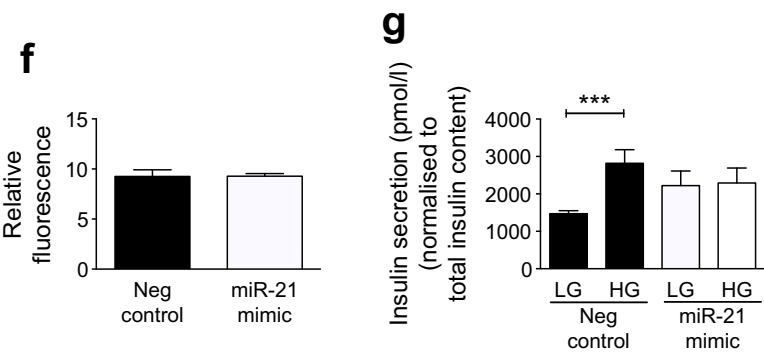

Fig. 2 miR-21 overexpression increases INS-1 beta cell apoptosis and decreases GSIS. INS-1 cells were transfected with a miR-21 mimic for $48 \mathrm{~h}$ to increase miR-21 activity. (a) Viability staining with AOPI, with green staining $(\mathrm{AO})$ representing all cells, and red staining (PI) representing compromised membranes. Scale bar, $400 \mu \mathrm{m}$. (b) Total cells and (c) percentage of dead cells were analysed. (d) Immunoblot for cleaved caspase-3. (e) Relative expression of ER stress transcripts was

from the polysome-associated fractions, indicating a reduction in active $B c l 2$ translation (Fig. 3i). These results suggest that both mRNA degradation and translational inhibition contribute to miR-21-mediated reductions in BCL2 protein levels.

To validate the relevancy of our findings in a human model, human islets were treated with a mix of proinflammatory cytokines for 24 and $48 \mathrm{~h}$. Similar to the results obtained in rodent beta cell lines and islets, we observed a significant increase in miR-21 expression at the $48 \mathrm{~h}$ time point (Fig. 4b). To confirm a direct role of miR-21 on BCL2 and apoptosis in human islets, we transfected miR-21 mimics into dispersed islets. Consistent with our findings above, we observed that miR-21 overexpression led to an increase in cleaved caspase 3 (measured using ELISA) and a reduction in $B C L 2$ expression (Fig. 4c, d).

Finally, to define effects of miR-21 inhibition, INS- 1 cells were transfected with a miR-21 inhibitor for $48 \mathrm{~h}$ (Fig. 5a, b). At baseline, a trend towards increased BCL2 levels was present (Fig. 5a), while PDCD4 levels were unchanged (Fig. 5b). To determine whether miR-21 inhibition had a protective effect against proinflammatory cytokines, INS- 1 cells were treated for $16 \mathrm{~h}$ with a proinflammatory cytokine mix. Interestingly, in combination with cytokine treatment, miR21 inhibition reduced cleaved caspase 3 levels compared with quantified with qPCR: black bars, control cells; white bars, mimic. (f) ROS generation was quantified using CellROX Deep Red Fluorescent Reagent. (g) After miR-21 mimic transfection, GSIS was assayed in response to low $(2.5 \mathrm{mmol} / \mathrm{l})$ and high $(15 \mathrm{mmol} / \mathrm{l})$ glucose concentrations, and normalised to the total insulin content of the cell lysate. Neg, negative. S/T Xbp1, spliced/total Xbp1. $n=4-9 . * p \leq 0.05 ; * * p \leq 0.01$, $* * * p \leq 0.001$

negative controls (Fig. 5c). Here, there was a trend towards increased BCL2 levels in cells pretreated with the miR-21 inhibitor (Fig. 5d). Again, no differences were present in PDCD4 levels (Fig. 5e).

In order to ascertain the contribution of BCL2 inhibition to the pro-death effects of miR-21, we transfected INS-1 828/33 cells, which constitutively overexpress $B c l 2$, with a miR-21 mimic [23]. BCL2 levels were decreased by the miR-21 mimic. However, BCL-2 production remained higher than levels observed in the wild-type INS-1 cells (Fig. 5f). Consistent with this, the percentage of dead cells (assessed by AOPI staining, quantified in Fig. $5 \mathrm{~g}$ ) was significantly lower in BCL2-overproducing INS-1 cells compared with wild-type INS-1 cells transfected with the mimic.

\section{Discussion}

Previous studies have demonstrated increased beta cell miR21 expression in response to cytokines and inflammation, specifically via activation of $\mathrm{NF} \kappa \mathrm{B}$ signalling $[13,15,19]$. Consistent with other cell types, beta cell miR-21 has been confirmed to target the tumour suppressor PDCD4 [15, 19]. Knockout of islet PDCD4 protected against beta cell death 
a

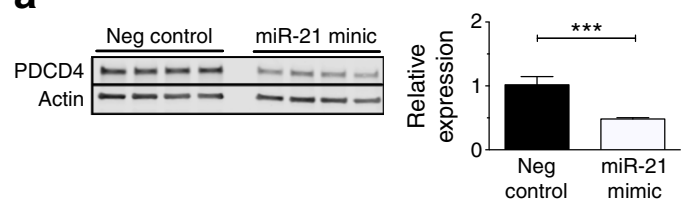

b

Position 705

BCL2 3'UTR: 5' ....agGAAAGUAUUUUUUUAAGCUa..

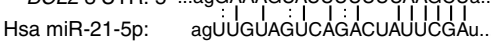

C

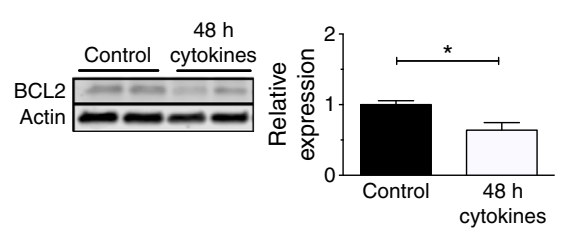

f
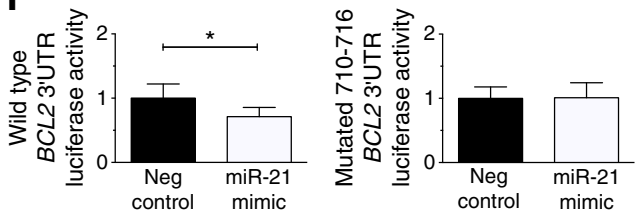

g
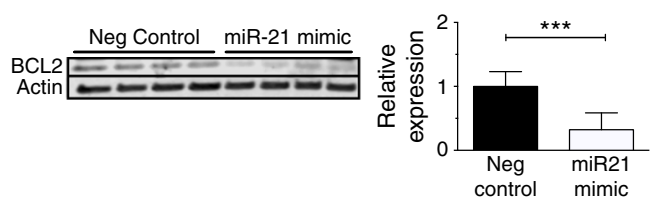

Fig. 3 miR-21 reduces BCL2 levels via directly targeting the BCL2 $3^{\prime}$ UTR, resulting in reduced mRNA levels and reduced translation of $B c l 2$ transcripts. (a) After miR-21 mimic transfection into INS-1 cells, immunoblotting was performed for PDCD4 $(n=4)$. (b) Predicted binding sites of miR-21 within BCL2 3' UTR. (c) Immunoblot for BCL2 after inflammatory cytokine treatment $(n=6)$. (d) $B c l 2$ mRNA levels after mimic transfection $(n=8)$. (e) mRNA expression of other BCL2 family members ( $n=4$; black bars, control; white bars, mimic). $B c l-X L$ is also known
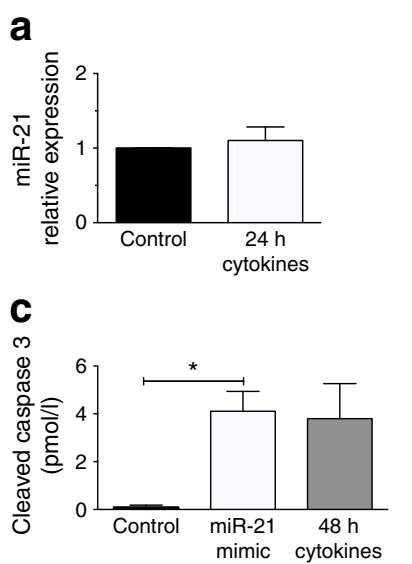

b

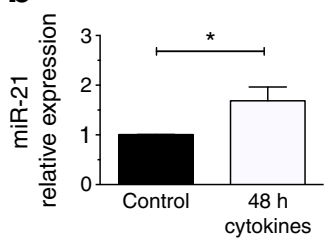

d

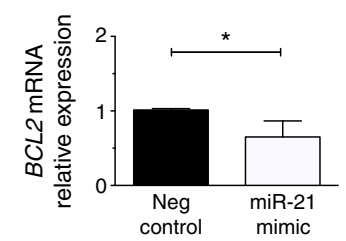

Fig. 4 Human islet miR-21 is increased by inflammatory cytokine treatment and increases apoptosis in association with reduced BCL2 expression. (a, b) miR-21 expression was quantified after human islets were treated with a cytokine mix for 24 and $48 \mathrm{~h}$. (c) Cleaved caspase 3 was quantified using ELISA in dispersed human islets after miR-21 mimic transfection. A $48 \mathrm{~h}$ treatment with cytokine mix was also included as a positive control. (d) $B C L 2$ transcripts were quantified after mimic transfection. Neg, negative. $n=3-4 . * p \leq 0.05$
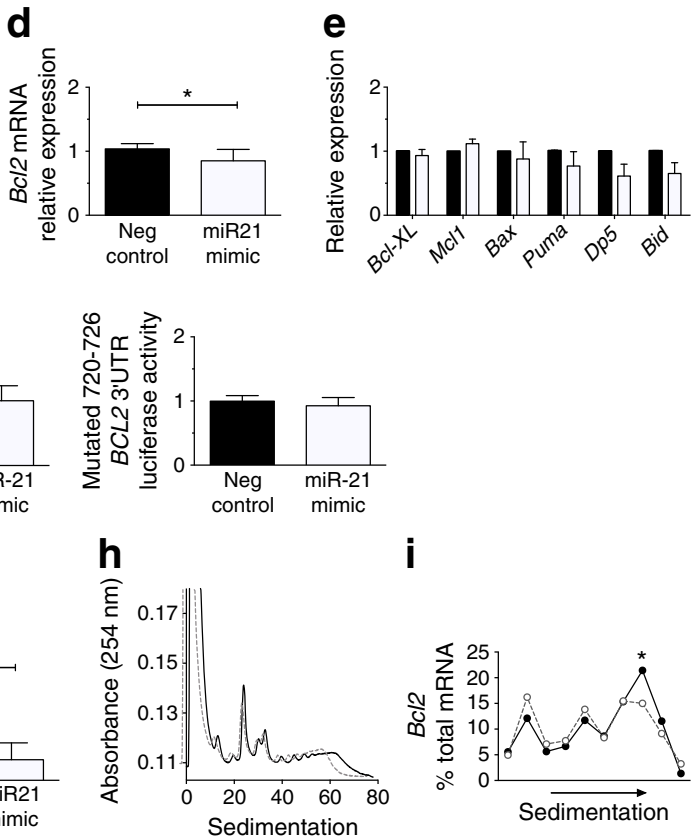

i

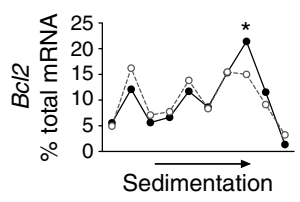

as Bcl2l1. (f) Luciferase assay was performed on INS-1 cells expressing wild-type or mutated BCL2 $3^{\prime}$ UTR and then transfected with a miR-21 mimic $(n=4)$. (g) Immunoblot for BCL2 after miR-21 mimic transfection $(n=8)$. (h, i) PRP analysis was performed after miR-21 mimic transfection $(n=3)$. Solid line, control; dashed line, mimic. (h) Representative global profile. (i) Aggregate data of individual profiles for $B c l 2$ mRNA. Neg, negative. $* p \leq 0.05 ; * * * p \leq 0.001$

and development of diabetes in NOD mice and after treatment with high-dose STZ, suggesting that miR-21-mediated inhibition of PDCD4 could potentially have a pro-survival effect [19]. However, others have shown that miR-21 overexpression via transfection demonstrated no improvement in beta cell survival [13]. More recent work reported increased beta cell apoptosis after stable lentiviral miR-21 overexpression. However, the authors did not observe concurrent reductions in PDCD4 with miR-21 overexpression, or identify a mechanism explaining the potential proapoptotic effects of miR-21 [14], leaving a number of unresolved controversies regarding the effect of miR-21 on beta cell survival and function.

Consistent with other studies, we found that beta cell miR21 was induced by multiple in vitro and in vivo models of inflammation and type 1 diabetes, including cytokine treatment of human islets, islets from mice treated with MLD-STZ, and NOD mouse islets. Moreover, we ruled out contributions from ER stress or hyperglycaemia associated with development of diabetes. The elevation of miR-21 in non-diabetic NOD islets is 

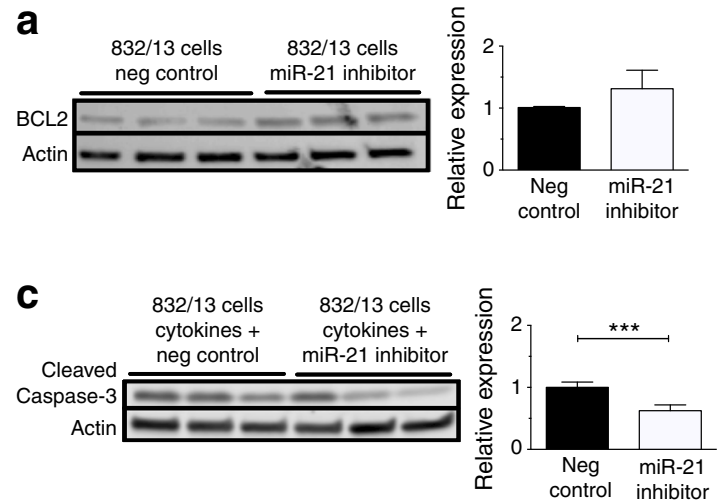

e
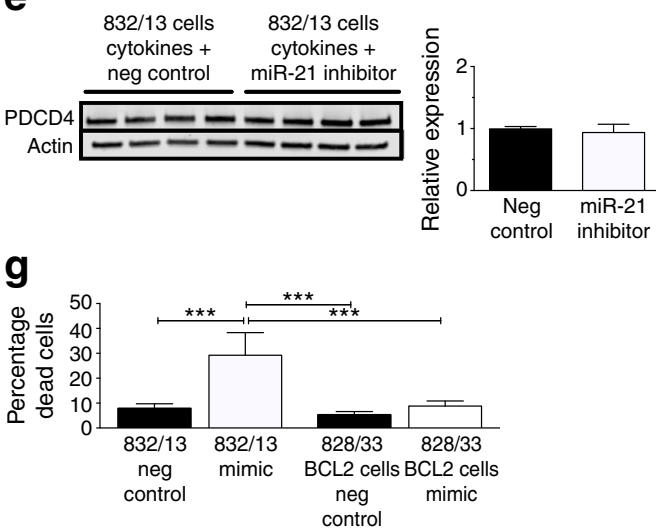

Fig. 5 miR-21 inhibition reduces cytokine-induced cleaved caspase 3 production, while BCL2 overexpression reduces miR-21-mediated apoptosis. (a, b) miR-21 LNA inhibitor was transfected into INS-1 832/13 (wild-type) beta cells for $48 \mathrm{~h}$ to inhibit miR-21 activity. Immunoblots for (a) BCL2 and (b) PDCD4 were performed. (c-e) After miR-21 inhibitor treatment, INS-1 832/13 cells were treated with $16 \mathrm{~h}$ of cytokine mix, and

an interesting finding, and is consistent with inflammatory induction of miR-21 expression, as NOD-severe combined immunodeficiency mice exhibit islet macrophage and dendritic cell infiltration, increased TNF- $\alpha$ signalling and intrinsic islet dysfunction, despite the absence of hyperglycaemia [32, 33]. Of note, and consistent with islet miR-21 expression, we were unable to detect differences in islet $B c l 2$ mRNA between diabetic and non-diabetic NOD mice (data not shown).

We endeavoured first to clarify the effects of miR-21 on beta cell survival, and second to identify a beta cell miR-21 target to explain these effects. In INS-1 beta cells, our results revealed that miR-21 overexpression, induced using a miR-21 mimic, led to a clear increase in cell death, despite reductions in PDCD4 protein levels. Correspondingly, inhibition of miR-21 was able to reduce cleaved caspase 3 production after cytokine treatment. Differences in our data and previous work may have several explanations, but are mostly likely due to differences in the method, dose and effectiveness of miR-21 manipulation in the target cells. However, we verified that our system resulted in effective miR-21 overexpression by ensuring significant suppression of PDCD4, a verified beta cell miR-21 target. Furthermore,
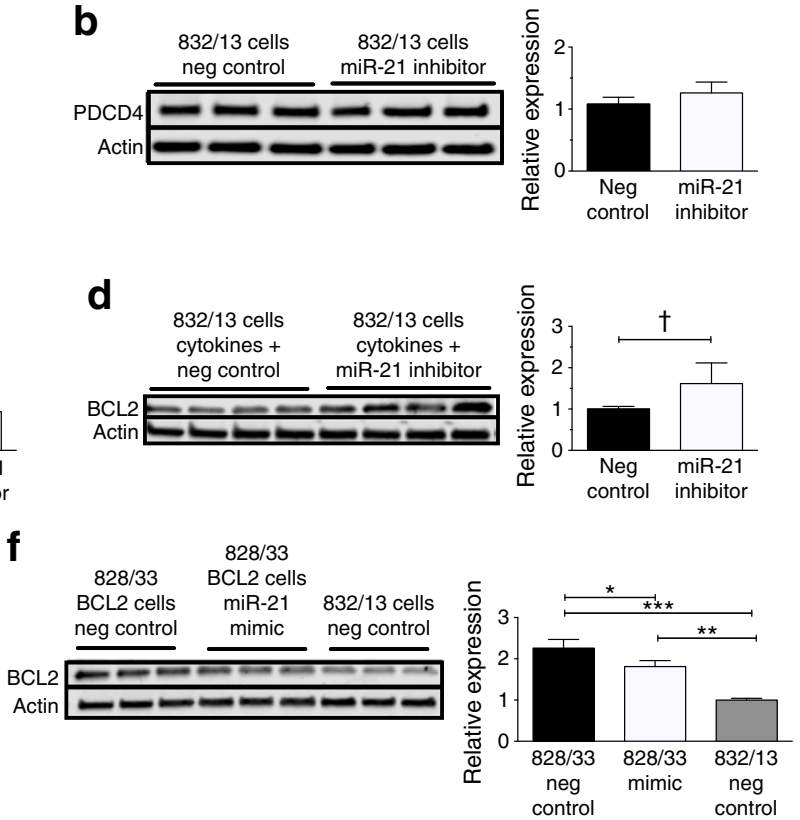

immunoblots were performed for (c) cleaved caspase 3, (d) BCL2, and (e) PDCD4. (f, g) INS-1 828/33 cells, which constitutively overexpress BCL2, were transfected with a miR-21 mimic for $48 \mathrm{~h}$. (f) Immunoblot for BCL2. (g) Percentage dead cells. Neg, negative. $n=3-5$. $* p \leq 0.05$, $* * p \leq 0.01, * * * p \leq 0.001,{ }^{\dagger} p=0.052$

identification of a target that explains the proapoptotic phenotype lends credibility to our system and findings.

Based on in silico prediction tools, we identified the $B C L 2$ $3^{\prime}$ UTR as a highly conserved miR-21 binding site. BCL2, a member of the antiapoptotic BCL2-like-protein subgroup of the BCL2 protein family, is involved in regulating the permeabilisation of the mitochondrial outer membrane and apoptosis [34]. When bound to BH3-only 'activator' proteins, BCL2-like proteins are able to render activator proteins latent. However, when the interaction between BCL2-like proteins and activator proteins is disrupted, activator proteins are released into the cytoplasm, where they induce a conformational change and activation of proapoptotic Bax-like proteins. This ultimately leads to pore formation in the outer mitochondrial membrane and release of proapoptotic proteins into the cytosol $[34,35]$. Because of BCL2's pro-survival properties, miR21-mediated reductions in the stability of BCL2 mRNA and suppression of $B C L 2$ translation could explain the proapoptotic phenotype we observed in beta cells $[36,37]$.

Although BCL2's 3' UTR has a predicted miR-21 binding site, reduced BCL2 levels have not been typically identified as 
an effect of miR-21. On the contrary, in other cell types, miR21 overexpression has been associated with increased BCL2 protein levels [38-40]. Treatment of breast cancer cells with oestrogen receptor agonists was associated with reductions of miR-21 expression and increased BCL2 mRNA and protein levels, suggesting a potential inhibitory interaction. However, these results could also be explained by a direct effect of oestrogen agonists on BCL2 production [41]. In contrast, our data support a direct interaction between miR-21 and the $B C L 23^{\prime}$ UTR in beta cells, and provide direct evidence of the effect of miR-21 on BCL2 mRNA levels as well as translation. Moreover, we confirmed the link between miR-21 overexpression, reductions in $B C L 2$ and increased apoptosis in human islets. This novel report of miR-21 inhibition of $B C L 2$ may explain the unusual proapoptotic phenotype of miR-21 seen in the beta cell. Indeed, overproduction of BCL2 was able to reduce miR-21-mediated death in INS-1 cells.

There are several limitations to our study. BCL2 overproduction has previously been shown to protect against cytokine-induced apoptosis in beta cell lines and islets ex vivo. However, in vivo BCL2 overproduction delayed but was unable to prevent the development of diabetes in NOD mice $[36,42,43]$. Although these data suggest that beta cell BCL2 overproduction may be partially protective, diabetes prevention in this model may require combinatorial approaches with a second intervention, such as immune modulation. Similarly, as previous work has suggested that loss of BCL2 in isolation may not be sufficient to induce apoptosis, key methodological differences in time frame and/or method of BCL2 reduction may explain our observed effects $[44,45]$.

Finally, BCL2 inhibition has recently been shown to increase beta cell mitochondrial metabolism and GSIS [44]. Because miR-21-induced reductions in BCL2 might be expected to increase GSIS, our findings of decreased GSIS with miR21 overexpression are not likely to be directly due to reduced BCL2 levels [44]. Our results are probably related to the progression of apoptosis and cell death present in a high percentage of cells, with subsequent degranulation and alterations in function. Alternatively, downregulation of other suggested miR-21 target mRNAs, such as $P c l o$, which encodes a protein important for cyclic AMP potentiation of insulin secretion, may lead to effects on insulin secretion independent of the effects of miR-21 on cell survival [15]. Further investigation of other miR-21 target mRNAs is needed to fully understand the in vivo relevance of changes in beta cell miR-21 expression.

In conclusion, our results suggest a novel role for miR-21 in the beta cell through the induction of apoptosis via $B C L 2$ mRNA degradation and inhibition of BCL2 mRNA translation. These findings provide an important insight into the role of inflammation-induced elevations in beta cell miR-21 during the development of diabetes. Future work will use in vivo beta cell-specific models to study the effects of miR-21 overand underexpression in order to define whether modulation of beta cell miR-21 levels might represent a potential target for strategies aimed at reducing beta cell death in diabetes.

Acknowledgements We acknowledge P. Fueger of Indiana University (Department of Pediatrics) and C. Newgard of Duke University, NC, USA (Departments of Medicine and Pharmacology and Cancer Biology) for their generous donation of INS-1 828/33 cells, and the Indiana University Center for Diabetes and Metabolic Diseases' Islet and Physiology Core for assistance with islet isolation. We also thank Y. Gu (Pediatrics, Indiana University) for technical assistance. Some of the data included in this manuscript were previously presented in abstract form at the Midwest Islet Club, the Combined Annual Meeting of the American Federation for Medical Research/Central Society for Clinical and Translational Research, the Pediatric Endocrine Society meeting, and the 76th Scientific Sessions of the American Diabetes Association, in 2016.

Data availability The datasets generated and/or analysed during the current study are available from the corresponding author on reasonable request.

Funding This manuscript was also supported by funding from NIDDK K08DK103983 to EKS, a Pediatric Endocrine Society Clinical Scholar Award to EKS, a pilot and feasibility award within the Center for Diabetes and Metabolic Diseases NIH/NIDDK Grant Number P30 DK097512, funding by Indiana University Health and the Indiana Clinical and Translational Sciences Institute to EKS, funded in part by Grant no. KL2TR001106 and Grant no. UL1TR001108, NIH Grant no. 32DK064466 to AJL, 1F32DK104501-01A1 to EA-B, and NIH grant R01 DK093954, VA Merit Award I01BX001733 and JDRF SRA-2014-41-Q-R (to CEM). This study used core services provided by the Diabetes Research Center grant P30 DK097512 to Indiana University School of Medicine.

Duality of interest The authors declare that there is no duality of interest associated with this manuscript.

Contribution statement EKS conceived and designed the experiments, acquired and analysed the data, and drafted and revised the manuscript. AJL acquired and analysed the data and revised the manuscript. EAB conceived the experiments, acquired and analysed the data, and revised the manuscript. TK conceived and designed the experiments, and revised the manuscript. XT contributed to concept and design, acquired and analysed the data, and revised the manuscript. CEM contributed to conception and design, interpreted/analysed the data, and drafted and revised the manuscript. All authors gave final approval of the version to be published. EKS is responsible for the integrity of the work as a whole.

\section{References}

1. Ameres SL, Zamore PD (2013) Diversifying microRNA sequence and function. Nat Rev Mol Cell Biol 14:475-488

2. Lynn FC, Skewes-Cox P, Kosaka Y, McManus MT, Harfe BD, German MS (2007) MicroRNA expression is required for pancreatic islet cell genesis in the mouse. Diabetes 56:2938-2945

3. Poy MN, Eliasson L, Krutzfeldt J et al (2004) A pancreatic isletspecific microRNA regulates insulin secretion. Nature 432:226-230

4. Jacovetti C, Abderrahmani A, Parnaud G et al (2012) MicroRNAs contribute to compensatory beta cell expansion during pregnancy and obesity. J Clin Invest 122:3541-3551

5. Latreille M, Hausser J, Stutzer I et al (2014) MicroRNA-7a regulates pancreatic beta cell function. J Clin Invest 124:2722-2735 
6. Osmai M, Osmai Y, Bang-Berthelsen CH et al (2016) MicroRNAs as regulators of beta-cell function and dysfunction. Diabetes Metab Res Rev 32:334-349

7. Kim KW, Ho A, Alshabee-Akil A et al (2016) Coxsackievirus B5 infection induces dysregulation of microRNAs predicted to target known type 1 diabetes risk genes in human pancreatic islets. Diabetes 65:996-1003

8. Tugay K, Guay C, Marques AC et al (2016) Role of microRNAs in the age-associated decline of pancreatic beta cell function in rat islets. Diabetologia 59:161-169

9. Filios SR, Shalev A (2015) Beta-cell microRNAs: small but powerful. Diabetes 64:3631-3644

10. Fernandez-Valverde SL, Taft RJ, Mattick JS (2011) MicroRNAs in beta-cell biology, insulin resistance, diabetes and its complications. Diabetes 60:1825-1831

11. Grieco FA, Sebastiani G, Juan-Mateu J et al (2017) MicroRNAs miR-23a-3p, miR-23b-3p and miR-149-5p regulate the expression of pro-apoptotic BH3-only proteins DP5 and PUMA in human pancreatic beta cells. Diabetes 66:100-112

12. Atkinson MA, Bluestone JA, Eisenbarth GS et al (2011) How does type 1 diabetes develop? The notion of homicide or beta-cell suicide revisited. Diabetes 60:1370-1379

13. Roggli E, Britan A, Gattesco S et al (2010) Involvement of microRNAs in the cytotoxic effects exerted by proinflammatory cytokines on pancreatic beta-cells. Diabetes 59:978-986

14. Backe MB, Novotny GW, Christensen DP, Grunnet LG, MandrupPoulsen T (2014) Altering beta-cell number through stable alteration of miR-21 and miR-34a expression. Islets 6:e27754

15. Bravo-Egana V, Rosero S, Klein D et al (2012) Inflammationmediated regulation of microRNA expression in transplanted pancreatic islets. J Transplant 2012:723614

16. Frampton AE, Castellano L, Colombo T et al (2015) Integrated molecular analysis to investigate the role of microRNAs in pancreatic tumour growth and progression. Lancet 385(Suppl 1):S37

17. Haghpanah V, Fallah P, Tavakoli R et al (2015) Antisense-miR-21 enhances differentiation/apoptosis and reduces cancer stemness state on anaplastic thyroid cancer. Tumour Biol 37:1299-1308

18. Wagenaar TR, Zabludoff S, Ahn SM et al (2015) Anti-miR-21 suppresses hepatocellular carcinoma growth via broad transcriptional network deregulation. Mol Cancer Res 13:1009-1021

19. Ruan Q, Wang T, Kameswaran V et al (2011) The microRNA-21PDCD4 axis prevents type 1 diabetes by blocking pancreatic beta cell death. Proc Natl Acad Sci U S A 108:12030-12035

20. Stull ND, Breite A, McCarthy R, Tersey SA, Mirmira RG (2012) Mouse islet of Langerhans isolation using a combination of purified collagenase and neutral protease. J Vis Exp : JoVE

21. Garber JC, Barbee RW, Bielitzki JT et al (2011) Guide for the care and use of laboratory animals, vol 8 . The National Academic Press, Washington DC, p 220

22. Hohmeier HE, Newgard CB (2004) Cell lines derived from pancreatic islets. Mol Cell Endocrinol 228:121-128

23. Tran VV, Chen G, Newgard CB, Hohmeier HE (2003) Discrete and complementary mechanisms of protection of beta-cells against cytokine-induced and oxidative damage achieved by bcl-2 overexpression and a cytokine selection strategy. Diabetes 52:1423-1432

24. Kono T, Ahn G, Moss DR et al (2012) PPAR-gamma activation restores pancreatic islet SERCA2 levels and prevents beta-cell dysfunction under conditions of hyperglycemic and cytokine stress. Mol Endocrinol 26:257-271

25. Chakrabarti SK, James JC, Mirmira RG (2002) Quantitative assessment of gene targeting in vitro and in vivo by the pancreatic transcription factor, Pdx1: importance of chromatin structure in directing promoter binding. J Biol Chem 277:13286-13293

26. Johnson JS, Kono T, Tong $X$ et al (2014) Pancreatic and duodenal homeobox protein $1(\mathrm{Pdx}-1)$ maintains endoplasmic reticulum calcium levels through transcriptional regulation of sarcoendoplasmic reticulum calcium ATPase $2 \mathrm{~b}$ (SERCA2b) in the islet beta cell. J Biol Chem 289:32798-32810

27. Sims EK, Hatanaka M, Morris DL et al (2013) Divergent compensatory responses to high-fat diet between C57BL6/J and C57BLKS/J inbred mouse strains. Am J Phys Endocrinol Metab 305:E1495-E1511

28. Hatanaka M, Maier B, Sims EK et al (2014) Palmitate induces mRNA translation and increases ER protein load in islet beta-cells via activation of the mammalian target of rapamycin pathway. Diabetes 63:3404-3415

29. Lewis BP, Burge CB, Bartel DP (2005) Conserved seed pairing, often flanked by adenosines, indicates that thousands of human genes are microRNA targets. Cell 120:15-20

30. Betel D, Wilson M, Gabow A, Marks DS, Sander C (2008) The microRNA.org resource: targets and expression. Nucleic Acids Res 36:D149-D153

31. Agarwal V, Bell GW, Nam J-W, Bartel DP (2015) Predicting effective microRNA target sites in mammalian mRNAs. elife 4:e05005

32. Dahlen E, Dawe K, Ohlsson L, Hedlund G (1998) Dendritic cells and macrophages are the first and major producers of TNF-alpha in pancreatic islets in the nonobese diabetic mouse. J Immunol 160: 3585-3593

33. Tersey SA, Nishiki Y, Templin AT et al (2012) Islet beta-cell endoplasmic reticulum stress precedes the onset of type 1 diabetes in the nonobese diabetic mouse model. Diabetes 61:818-827

34. Martinou JC, Youle RJ (2011) Mitochondria in apoptosis: Bcl-2 family members and mitochondrial dynamics. Dev Cell 21:92-101

35. Gurzov EN, Eizirik DL (2011) Bcl-2 proteins in diabetes: mitochondrial pathways of beta-cell death and dysfunction. Trends Cell Biol 21:424-431

36. Iwahashi H, Hanafusa T, Eguchi Y et al (1996) Cytokine-induced apoptotic cell death in a mouse pancreatic beta-cell line: inhibition by Bcl-2. Diabetologia 39:530-536

37. Rabinovitch A, Suarez-Pinzon W, Strynadka K et al (1999) Transfection of human pancreatic islets with an anti-apoptotic gene (bcl-2) protects beta-cells from cytokine-induced destruction. Diabetes 48:1223-1229

38. Li Y, Yan L, Zhang W et al (2014) miR-21 inhibitor suppresses proliferation and migration of nasopharyngeal carcinoma cells through down-regulation of BCL2 expression. Int J Clin Exp Pathol 7:3478-3487

39. Dong J, Zhao Y-P, Zhou L, Zhang T-P, Chen G (2011) Bcl-2 upregulation induced by miR-21 via a direct interaction is associated with apoptosis and chemoresistance in MIA $\mathrm{PaCa}-2$ pancreatic cancer cells. Arch Med Res 42:8-14

40. Si M, Zhu S, Wu H, Lu Z, Wu F, Mo Y (2007) miR-21-mediated tumor growth. Oncogene 26:2799-2803

41. Wickramasinghe NS, Manavalan TT, Dougherty SM, Riggs KA, Li Y, Klinge CM (2009) Estradiol downregulates miR-21 expression and increases miR-21 target gene expression in MCF-7 breast cancer cells. Nucleic Acids Res 37:2584-2595

42. Allison J, Thomas H, Beck D et al (2000) Transgenic overexpression of human Bcl-2 in islet beta cells inhibits apoptosis but does not prevent autoimmune destruction. Int Immunol 12:9-17

43. Liu Y, Rabinovitch A, Suarez-Pinzon W et al (1996) Expression of the bcl-2 gene from a defective HSV-1 amplicon vector protects pancreatic beta-cells from apoptosis. Hum Gene Ther 7:1719-1726

44. Luciani DS, White SA, Widenmaier SB et al (2013) Bcl-2 and Bcl$\mathrm{xL}$ suppress glucose signaling in pancreatic beta-cells. Diabetes 62 : $170-182$

45. Aharoni-Simon M, Shumiatcher R, Yeung A et al (2016) Bcl-2 regulates reactive oxygen species signaling and a redox-sensitive mitochondrial proton leak in mouse pancreatic $\beta$-cells. Endocrinology 157:2270-2281 